\title{
Lamartine : les deux vices du gouvernement temporel de la papauté dans l'article du 28 octobre 1847
}

\author{
Jean-Baptiste JEANGENE VILMER \\ Université Paris-IV Sorbonne \\ $\rightarrow$ publié dans Revue d'Histoire Ecclésiastique, 99:3-4, 2004, p. 627-665 \\ cet article est mis à la disposition du lecteur mais il ne correspond pas à la mise en page de la version \\ définitive et publiée à laquelle il convient de se référer pour toute citation
}

\begin{abstract}
RÉSUMÉ - En juin 1846, dans l'Italie divisée du Congrès de Vienne, l'élection de Pie IX donne au néoguelfisme une occasion de poser à nouveaux frais la question de l'unité italienne. Le 28 octobre 1847, à la veille des révolutions romaine et parisienne, Alphonse de Lamartine, qui entretient avec la religion des rapports complexes et ambivalents, rejette alors la solution néo-guelfe et se justifie par une longue et intense critique du pouvoir temporel de la papauté. A travers l'analyse détaillée de la critique lamartinienne, et dans le cadre de l'histoire de la curie romaine depuis la victoire française en Italie jusqu'à l'avènement de Pie IX (1796-1846), nous examinons ce que Lamartine présente comme les deux vices du gouvernement pontifical : sa complexité et son instabilité, c'est-à-dire, en somme, les questions de sa nature et de la continuité de son pouvoir.
\end{abstract}

ABSTRACT - In June 1846, in an Italy divided by the Congress of Vienna, the election of Pius IX gives neo-Guelfists an opportunity to reconsider the question of Italian unity. In October 1847, on the eve of Roman and Parisian revolutions, Alphonse de Lamartine, who has complex and ambivalent relationships with religion, rejects the neo-Guelfist solution and justifies himself with a long and intense criticism of the temporel power of the papacy. Through the detailed analysis of Lamartinian criticism, and within the scope of the history of Roman Curia from the French victory in Italy to the accession of Pius IX (1796-1846), I examine what Lamartine presents as the two vices of the papal government: its complexity and its instability - that is to say, the questions of its nature and of the continuity of its power.

ZUSAMMENFASSEN - Im Juni 1846, Italien geteilt durch den Kongreß Wiens, gibt die Wahl von Pius IX dem neo-Guelfismus eine Gelegenheit, die Frage nach den Kosten der italienischen Einheit zu stellen. Am 28. Oktober 1847, kurz vor den römischen und Pariser Revolutionen, weist Alphonse de Lamartine, der komplizierte und ambivalente Beziehungen zur Religion hat, die neo-guelfistische Lösung zurück und rechtfertigt sich mit einer langen und starken Kritik an der zeitlichen Macht des Papsttums. Durch die ausführliche Analyse der Lamartinischen Kritik und im Rahmen der Geschichte der römischen Kurie seit dem französischen Sieg in Italien bis zur Thronbesteigung von Pius IX ( 1796-1846 ), prüfen wir, was Lamartine als die beiden Laster der päpstlichen Regierung darstellt : ihre Kompliziertheit und ihre Unbeständigkeit, das heißt insgesamt, die Fragen ihrer Natur und der Kontinuität ihrer Macht. 
Alphonse de Lamartine entretient avec la religion un rapport constant, instable et complexe. Après une éducation catholique et des études chez les jésuites à Belley, il rompt avec la foi à dix-huit ans lorsqu'il découvre en automne 1808 les philosophes du XVIIIe siècle chez son ami Guichard, puis y revient pour son mariage en 1820. Il l'égare à nouveau en 1832, année de rupture, pour deux raisons : un voyage en terre sainte le convainc de l'immatérialité de Dieu, donc de la seule humanité du Christ (son Voyage en Orient de 1836 sera mis à l'Index par l'Église) et, le 7 décembre, il perd sa fille Julia. La rupture avec la foi chrétienne est définitive, mais une présence souterraine se profile encore derrière chacun de ses écrits : ce que Vigny dit, en 1834, du christianisme, à savoir qu'il est « un caméléon éternel » ${ }^{1}$, Lamartine l'incarne à merveille. Sa religion sera désormais un « christianisme rationnel » ou un " rationalisme chrétien », comme il le dit lui-même ${ }^{2}$. A l'occasion de son voyage de 1844, et alors qu'il prévoyait de se rendre au Vatican, il écrit dans son carnet un projet : «St. Pierre, transformation du catholicisme en religion rationnelle, essai $»^{3}$. Sa position semble être un déisme dix-huitiémiste digne de son maître Voltaire. On en trouve un exemple en 1846, dans le discours que l'abbé Thions fait rédiger par Lamartine pour faire connaître à ses paroissiens qu'il quitte l'Église, n'ayant plus la foi - un discours dans lequel l'auteur s'attaque aux « dogmes obscurs » et aux « institutions vermoulues » du christianisme, avant de faire un éloge de Voltaire ${ }^{4}$.

Ce faisant, il prend de la distance avec la papauté et porte un regard très critique sur le gouvernement temporel ${ }^{5}$. En 1833, il aide ceux « qui s'étaient jetés hors de l'Église du pape » en prenant parti, à la Chambre, pour le droit d'association en raison d'un « besoin religieux ». Et, en 1836, dans Jocelyn, très sévèrement critiqué par la presse catholique et mis à l'Index par décret du 22 septembre de la même année ${ }^{6}$, il condamne les ambitions temporelles de l'Église, dénonce les papes de la Rome moderne et a des vers sur le déclin de la religion romaine, thème favori des libéraux. Le texte le plus complet, le plus dense et le plus explicite témoignant de la critique lamartinienne du pouvoir temporel de la papauté se trouve dans l'article du 28 octobre 1847, consacré à « La situation de la France à l'extérieur en 1847 », en l'occurrence à l'Italie, que l'auteur fait paraître dans $L e$ Bien public, un bihebdomadaire qu'il publie à Mâcon.

$\mathrm{Au}$ sein même de l'article, le passage concerné s'avère être la mineure d'un syllogisme qui, résumé, se développe de la façon suivante : Il faut que les États italiens s'unissent en une Confédération. Or, on ne peut confier la responsabilité de ce changement à Rome, car ce gouvernement est très imparfait. Donc, c'est à la France de

\footnotetext{
${ }^{1}$ Alfred de VIGNY, Le Journal d'un poète, F. Baldenspergered (ed.), Paris, Conard, 1935, p. 288.

${ }^{2}$ Sous le nom d'abbé Thyons, dans une lettre au journal l'Univers en novembre 1846.

${ }^{3}$ Cité par Robert MATTLÉ, Lamartine voyageur, Thèse présentée à la Faculté des Lettres de l'Université de Fribourg (Suisse) pour obtenir le grade de docteur, Paris, E. de Boccard, p. 129, n. 6.

${ }^{4}$ Voir Henri GUILLEMIN, Lamartine, Paris, Seuil, 1987, p. 133, qui cite le texte du manuscrit.

${ }^{5}$ La distance qu'il prend avec la papauté tout en continuant à avoir une certaine relation avec le christianisme pose à l'interprète la question de la relation de Lamartine avec le protestantisme : une relation complexe, non assumée, mais présente comme le montre M. MAURICE, «Lamartine et le protestantisme ", in Actes du cinquantième Congrès de l'Association Bourguignonne des Sociétés Savantes, Tome I : Quatrièmes journées d'études Lamartiniennes, 25 au 27 mai 1979, Mâcon, Association Bourguignonne des Sociétés Savantes, p. 43-52.

${ }^{6}$ Dans son discours du 20 septembre 1847, il répondait encore à l'accusation de panthéisme dont il avait hérité avec Jocelyn, neuf années auparavant.
} 
jouer le rôle de médiateur armé pour organiser la confédération italienne. On comprend ainsi que la critique lamartinienne du pouvoir temporel de la papauté émerge à l'occasion du rejet de la solution néo-guelfe. Cet aspect, qui relève de l'histoire diplomatique, ne sera pas abordé ici : nous y avons consacré une étude indépendante sur Lamartine et Pie $\mathrm{IX}^{7}$, dont le présent article constitue en quelque sorte la seconde partie. Il s'agit maintenant, sur le plan cette fois de l'histoire ecclésiastique, de détailler le contenu de la critique lamartinienne du gouvernement temporel de la papauté, à travers l'analyse de l'extrait concerné de l'article du 28 octobre $1847^{8}$.

Comment procède l'auteur, dans ces lignes? L'introduction présente la thèse défendue, à savoir que le gouvernement pontifical a «les vices de toutes les natures de gouvernement, sans leurs avantages, réunis dans un seul gouvernement $»^{9}$. Conformément au découpage grammatical de cette affirmation en deux propositions, nous procéderons en deux parties. Un premier moment a pour objet d'établir que ce gouvernement pontifical a « les vices de toutes les natures de gouvernement », autrement dit qu'il est vicié par sa complexité. Un second moment s'attache ensuite au versant complémentaire de la thèse, à savoir que le gouvernement pontifical, qui donc a les vices de toutes les natures de gouvernement, n'a cependant aucun de leurs avantages. La raison invoquée est qu'il est instable, qu'il est donc vicié par son instabilité. La complexité et l'instabilité sont, pour Lamartine le 28 octobre 1847, les deux vices du gouvernement temporel de la papauté.

\section{I- Un gouvernement vicié par sa complexité}

La complexité du gouvernement pontifical surgit dans la question de sa nature : de quelle sorte de gouvernement s'agit-il ? A l'aune de la taxinomie habituelle, la réponse est loin d'être évidente pour Lamartine :

« Le gouvernement de la papauté temporelle à Rome est tout à la fois une théocratie ou le gouvernement d'un pontificat éternel, une oligarchie ou le gouvernement d'un petit groupe d'hommes influents dans l'État, les cardinaux; une monarchie, car le Pape est roi ; une république, car les chefs de l'oligarchie sacerdotale l'élisent et le déposent ; une aristocratie, car les princes romains sont les vassaux du pape et les suzerains du peuple; une démocratie, car l'élection y est le principe de la souveraineté $(\ldots) »^{10}$

La difficulté pour le commentateur est la tâche qui lui revient, par défaut, de regrouper et classer les six sortes de régimes arbitrairement énumérées par l'auteur. Nous proposons de les analyser en deux temps, selon la dialectique classique de l'un et du multiple, le multiple venant limiter l'un. Après avoir vu le gouvernement pontifical en tant que gouvernement de l'Un (monarchie, théocratie), nous verrons comment l'absoluité qui pourrait, en droit, en découler est de fait limitée par sa nature de gouvernement multiple :

\footnotetext{
${ }^{7}$ Voir notre «Lamartine et Pie IX : la France face à la question nationale italienne en 1846-1848 », à paraître.

${ }^{8}$ L'extrait en question se trouve dans l'article du 28 octobre 1847, in La France parlementaire (18341851), cuvres oratoires et écrits politiques, précédés d'une étude sur la vie et les œuvres de Lamartine par Louis Ulbach, Paris, 1864-1865, vol. 5, p. 93-94.

${ }^{9}$ Aphonse de LAMARTINE, article du 28 octobre 1847 [voir n. 8], p. 93.

${ }^{10}$ Ibid.
} 
JB Jeangène Vilmer, « Lamartine : les deux... », Revue d'Histoire Ecclésiastique, 2004

gouvernement de quelques-uns (oligarchie et aristocratie) et gouvernement élu (république et démocratie).

\title{
§1. UE gouverEemeEt complexe, qui est « tout à la fois » : l’UE et le Multiple
}

\section{A/ L'UE (théocratie et moEarchie)}

\begin{abstract}
"(...) il est impossible de nier que les papes soient des souverains, soit en vertu d'un mandat de Dieu, soit en vertu d'une antique tradition humaine (...) $\|^{11}$
\end{abstract}

La théo-cratie, qui étymologiquement associe Dieu au pouvoir, est une forme de gouvernement dans laquelle l'autorité est exercée par les représentants de Dieu et en son nom. Tirant son origine de l'expression fameuse de saint Paul selon laquelle il n'est de pouvoir sinon de Dieu, nisi potestas a Deo ${ }^{12}$, l'idée d'une théocratie apparaît dans la Bible à travers celle d'une " cité de Dieu », bientôt reprise par saint Augustin dans son De Civitate Dei. Idée ancienne, donc, et très vaste. En quel sens Lamartine l'entend-elle?

La précision « ou le gouvernement d'un pontificat éternel » nous engage d'emblée sur le terrain d'une théocratie précisée pontificale. La théocratie pontificale, distincte de la théocratie royale ou impériale instituée par Charlemagne, et selon laquelle le représentant de Dieu sur terre était l'Empereur lui-même, apparaît avec la réforme grégorienne, c'està-dire dans les vingt-sept propositions du Dictatus Papae de 1075 : c'est alors le pape, en l'occurrence Grégoire VII (1073-1085), qui représente sur terre le pouvoir divin. Il n'est plus le vicaire de Pierre, mais directement celui du Christ ${ }^{13}$. Sa vocation n'est plus seulement spirituelle, elle est également temporelle. Son autorité s'exerce dans sa plénitude (plenitudo potestatis). Le courant théocratique se développera dans l'Eglise pour atteindre son apogée, semble-t-il, avec la bulle Unam sanctam que Boniface VIII promulgue en 1302 à l'occasion de son opposition à Philippe IV le Bel. Il ne cessera ensuite de décroître, au profit peut-être de la théorie de la monarchie pontificale, qui lui est proche comme en témoigne la communauté de pensée que se partagent déjà, à la même époque, les représentants du courant théocratique, tel Gilles de Rome, dans son $D e$ eccleslastica potestate de 1301 et ceux du courant monarchique, tel Jacques de Viterbe, dans son De regimine christiano de 1302. La transition de la théocratie à la monarchie semble donc toute trouvée : n'est-elle pas historique ?

Toute l'importance de l'affirmation de Lamartine, selon laquelle le gouvernement pontifical serait une théocratie définie comme «le gouvernement d'un pontificat éternel », réside dans la compréhension de ce dernier mot: en quoi le pontificat est-il « éternel » ? On peut l'entendre en deux sens, qu'il est fécond de distinguer.

Premièrement, au sens littéral. Comme le rappellera cinq années plus tard A. Comte

\footnotetext{
${ }^{11}$ Alphonse de LAMARTINE, Cours familier de littérature : un entretien par mois, Paris, F. Didot, 18561866, vol. 19, 1865, p. 5-6.

${ }^{12} \mathrm{Rm}, 13,1$.

${ }^{13}$ « Tu es Pierre et sur cette pierre je bâtirai mon Église. (...) Je te donnerai les clefs du royaume des cieux ; tout ce que tu lieras sur la terre sera lié dans les cieux, et tout ce que tu délieras sur la terre sera délié dans les cieux » (Matthieu, XVI, 18-19). Voir J. GAUDEMET, Église et cité : histoire du droit canonique, Paris, Cerf, Montchrestien.
} 
dans son Catéchisme positiviste (1852), «La théocratie [...] repose sur deux institutions connexes, l'hérédité des professions quelconques, et l'universelle prépondérance de la caste sacerdotale $\gg{ }^{14}$. Autrement dit, le pontificat est éternel et il s'agit alors d'une théocratie en ce sens qu'il se transmet systématiquement, « héréditairement » pourrait-on dire en termes d'hérédité de classes et non de gènes, de la caste sacerdotale à elle-même. Le gouvernement est éternel car le temps ne saurait ni l'user ni en modifier l' " hérédité ». La critique sous-jacente consisterait alors à le dire incapable de changement, c'est-à-dire littéralement anti-moderniste, dans la structure même de son transfert de souveraineté.

Deuxièmement, au sens catholique. N'est-il pas paradoxal, après avoir précisé que l'on ne considérait ici que le "gouvernement temporel $»^{15}$ de le définir comme un «pontificat éternel»? La confusion est toute intentionnelle, et la critique de Lamartine est dissimulée à cet endroit: l'accusation de théocratie est celle de la confusion des pouvoirs temporel et spirituel. Dire que le gouvernement pontifical, «considéré comme un gouvernement temporel», est une théocratie, c'est-à-dire «le gouvernement d'un pontificat éternel ", c'est dire qu'un tel gouvernement confond ses rôles temporel et spirituel. Mêler les deux, dit Lamartine, c'est faire du gouvernement temporel une théocratie. L'auteur se prononce donc implicitement pour une séparation plus claire entre les pouvoirs temporel et spirituel de la papauté. Ce faisant, il se fait le porte-parole discret d'une opinion largement répandue à l'époque et qui ne cessera de gagner du terrain jusqu'à l'abolition du pouvoir temporel, en 1870 : «L'Italie reconnaît avec respect dans Pie IX le prêtre du Christ et non plus le prince temporel [...]. Le lambeau de la robe nazaréenne et le lambeau du manteau princier sont deux morceaux d'étoffe qui désormais ne peuvent plus se coudrent ensemble $»^{16}$. Cette confusion des pouvoirs dénoncée par Lamartine et nombre de ses contemporains était déjà écrite en vers dans la description de Rome que livre Voltaire au chant IV de sa Henriade, un texte dans la lignée duquel notre auteur revendique sa place :

\footnotetext{
« Le trône est sur l'autel, et l'absolu pouvoir Met dans les mêmes mains le sceptre et l'encensoir. " ${ }^{17}$
}

La mon-archie désigne littéralement l'État commandé par un seul : le monarque. Celui-ci est un organe individuel non représentatif, et généralement héréditaire ou nommé à vie, mais qui peut également être élu. En ce sens large, la papauté - dont le mot en français fut d'ailleurs créé au XIVe siècle sur le modèle de "royauté » et " principauté » - est effectivement une monarchie. Lapo da Castiglionchio parle au milieu du XVe siècle de « monarchie du Christ» pour la désigner, et la compare notamment avec Athènes. Le

\footnotetext{
${ }^{14}$ Auguste COMTE, Catéchisme positiviste, 11e entretien, Pécaut (ed.), Paris, Garnier, 1909, p. 344-345.

${ }^{15}$ Lamartine introduit effectivement son texte en prévenant que le gouvernement de la papauté à Rome sera « considéré comme gouvernement temporel» (article du 28 cotobre 1847 [voir n. 8], p. 93).

${ }^{16}$ Antoine JACQUEMOUD à G.-B. Michelini le 15 décembre 1848, in Atti del Parlemento subalpino. Sessione 1848. Camera dei deputati. Discussioni, Turin, 1856, p. 1165-1166. En 1860, Lamartine développera cette idée que "Les papes, humainement considérés, sont une dualité dans un même homme» : voir Cours familier [voir n. 11], vol. 9, 1860, p. 445.

${ }^{17}$ VOLTAIRE, La Henriade, chant IV, Danièle Thomas (ed.), Oloron-Sainte-Marie, Monhélios, 2002, p. 57. On trouve une critique de la théocratie au chapitre IX de l'Introduction à son Essai sur les moeurs.
} 
pape est un roi, l'image est classique. A l'époque de Lamartine, qui écrira en 1865 que «Les papes ont donc comme souverains un gouvernement $»^{18}$, les expressions de gouvernement « monarchico-clérical » et d' " hybride du pape-roi », sont courantes. Le rejet régulier de l'idée d'un «gouvernement des prêtres » durant cette période conforte d'ailleurs l'idée que le pape devait régner et non gouverner. Reste que cette conception est également crépusculaire : Pie IX est le dernier « pape-roi » (« Il Papa Re »). Si le pape est un roi, il devient effectivement de plus en plus un roi sans royaume, au fur et à mesure que fond son pouvoir temporel pour finalement disparaître en 1870 .

Le genre n'est certes pas contestable: le gouvernement pontifical est, de fait, une monarchie, car le pape est un roi. Plus difficile est la question de l'espèce : de quelle sorte de monarchie s'agit-il ? D'une espèce rare, pour commencer, car elle est élective (Lamartine parle de " cette monarchie élective $\|^{19}$ ) : le pape-roi est en effet élu par les cardinaux. Ensuite, la monarchie pontificale est-elle absolue ou limitée ? Et, le cas échéant, par quoi est-elle limitée?

La monarchie pontificale absolue a une réalité historique, qui prit un nouvel élan avec Innocent IV (1243-1254) et les théoriciens qui l'entouraient. Elle signifie que le pape n'est plus soumis qu'au droit divin : il n'est plus soumis au droit ecclésial et peut abolir les lois de l'Église à tout moment. Il y a, en somme, primauté du pape sur le droit ecclésial positif. Cette idée est développée par les théoriciens du début du XIVe siècle, tels Gilles de Rome et Agostino Trionfo : le pape est l'Église, «papa, qui potest dici ecclesia », ou l'Église est le « corps du pape ».

Qu'en est-il au XIXe siècle ? L'absoluité de la monarchie pontificale n'est bien entendu plus au goût du jour mais, après les désordres laissés par les guerres napoléoniennes, la papauté et l'infaillibilité apparaissent comme des garanties séduisantes de retour à l'ordre, et l'idée de la suprématie du pape, sur laquelle insiste Joseph de Maistre dans Du Pape en 1819, connaît un certain succès. Peut être dite monarchie absolue celle dans laquelle le roi possède tous les pouvoirs, prend et assume toutes les décisions, que ce soit directement ou indirectement. Poser la question de l'absoluité de la monarchie pontificale, c'est donc demander: le pape possède-t-il tous les pouvoirs ? Prend-il et assume-t-il toutes les décisions ? Certes non. Sa monarchie est limitée par trois éléments : la tradition, les organes collégiaux (ce que Lamartine nomme " oligarchie ») et les constitutions, ou du moins le mouvement constitutionnaliste des années 1846-1848. Ces trois éléments seront développés plus bas.

\section{B/ Le Multiple}

\section{1) Le gouverEemeEt de quelques-uEs (oligarchie et aristocratie)}

L'oligarchie est un système politique dans lequel la souveraineté est détenue par un petit nombre (« quelques-uns » dit le grec) d'individus ou de familles. Lamartine précise que ces oligarques, en l'occurrence, sont les cardinaux. Il n'y a rien à corriger : il a tout à fait raison. La domination des cardinaux est structurelle, depuis les origines de la curie romaine. Elle est même double : une domination collégiale et individuelle. D'une part, le

\footnotetext{
${ }^{18}$ Alphonse de LAMARTINE, Cours familier [voir n. 11], vol. 19, 1865, p. 7.

${ }^{19}$ Aphonse de LAMARTINE, article du 28 octobre 1847 [voir n. 8], p. 93.
} 
pouvoir des cardinaux s'affirme à travers des organes collégiaux dont le pouvoir de décision est déterminant. Ainsi en est-il du Sacré Collège, qui regroupe 1'ensemble des cardinaux, répartis en trois ordres (cardinaux-évêques, cardinaux-prêtres et cardinauxdiacres), qui ont le privilège d'élire collégialement le pape en conclave, d'administrer les affaires courantes en cas de vacance du Siège apostolique, et d'assister le pape dans l'administration de l'Église au sein des congrégations et dicastères de la Curie. Le rôle de conseil des cardinaux est perpétué et répandu dans la création d'autres organes collégiaux, comme la congrégation des affaires ecclésiastiques extraordinaires (Congregatio extraordinaria praeposita negotiis ecclesiasticis orbis catholici) de 1814, composée de huit cardinaux, d'un secrétaire et de cinq consulteurs, qui rendait des avis pour orienter et guider les décisions du pape. P. Rossi, dans une lettre à Guizot du 20 avril 1832, dénonce même le caractère oligarchique de certains dicastères du gouvernement central de l'Église : le Saint Office et l'Index, par exemple, sont « au service de toutes les oligarchies $\gg{ }^{20}$.

Si le collège cardinalice s'affirme progressivement comme Sénat de l'Église, et que les pouvoirs juridictionnels et politiques des cardinaux se renforcent, cette domination reste cependant indépendante du pouvoir collégial : elle est également individuelle. L'histoire du gouvernement pontifical montre que les papes ont toujours cherché à réduire le pouvoir du Collège des cardinaux, sans parvenir pour autant à affaiblir le pouvoir individuel des cardinaux en question. Cette domination résiste effectivement au déclin du Collège à l'époque du Grand Schisme d'Occident (1378-1417), à la constitution Immensa Aeterni Dei du 22 janvier 1588 de Sixt-Quint, qui visait pourtant à réduire l'influence du consistoire, comme à Benoît XIV (1740-1758), " pape des Lumières », qui le consultait peu. Le Collège a beau décliner, le pouvoir individuel des cardinaux, comme conseillers du pape, persiste.

L'oligarchie apparaît finalement comme une limitation de l'absoluité de la monarchie pontificale. Au point que certains en font même un argument en faveur de la non responsabilité du pape ${ }^{21}$.

L'aristocratie, littéralement «le pouvoir aux meilleurs», est un système de gouvernement dans lequel le pouvoir politique est exercé exclusivement par une minorité qui se considère comme l'élite de la société. La définition qu'en donne Lamartine (« car les princes romains sont les vassaux du pape et les suzerains du peuple »), nous semble relativement inadéquate. D'une part, la structure «peuple < princes < pape » ne met effectivement pas en évidence que le pouvoir est exercé exclusivement par une minorité, puisqu'elle reconnaît qu'il l'est en fin de compte par un organe individuel : le pape. S'il s'agit d'une aristocratie, elle s'inscrit donc dans le cadre d'une monarchie avec laquelle elle n'est par conséquent pas incompatible. D'autre part, il faudrait montrer que les princes se considèrent comme étant «les meilleurs ». Mais le propos de Lamartine n'est pas tant de faire une utilisation correcte de l' « aristocratie » entendue étroitement que de

\footnotetext{
${ }^{20}$ Pellegrino ROSSI «Sur 1'histoire de France sous Napoléon », Mélanges d'économie politique, de politique, d'histoire et de philosophie, Paris, Guillaumin, vol. 2, 1867, p. 238.

21 Voir le rapport d'Auguste De Liedekerke de Beaufort du 28 juillet 1846, cité par François JANKOWIAK, L'évolution des structures de la curie romaine de l'avènement de Pie IX à la fin du pontificat de Pie X (1846-1914), Thèse d'histoire du droit sous la direction de Brigitte BasdevantGaudemet, Université Paris-XI Orsay, 2002, p. 109.
} 
s'en servir, quitte à le faire vaguement, pour défendre des thèses fortes. On peut effectivement déduire au moins deux prises de position.

Premièrement, le choix terminologique ( princes », " vassaux », « suzerains ») et la hiérarchie pyramidale «peuple < princes < pape » désignent très nettement la féodalité. L'apparition de la féodalité, dès le IXe siècle en Europe occidentale et notamment en France, étant due en partie à la dissolution de la propriété foncière, cette référence nous place d'emblée sur le terrain, très temporel car matériel, de l'État de l'Église non plus comme gouvernement mais comme territoire, en l'occurrence découpé en fiefs. Le lien de vassalité, qui attache l'homme libre à un suzerain par un serment, structurerait donc, selon l'auteur, le gouvernement pontifical de la manière suivante : le peuple est le vassal des princes romains qui sont les vassaux du pape. Dans la structure féodale, c'était par l'intermédiaire du seigneur que l'homme libre obéissait au roi. En l'espèce, est-ce par l'intermédiaire du prince romain que le peuple obéit au pape? Certes non, le gouvernement pontifical s'adresse directement au peuple en ce qu'il est aussi spirituel : c'est la confusion des pouvoirs temporel et spirituel, évoquée précédemment, qui ici exclut qu'il y ait un lien de vassalité entre le peuple, le prince romain et le pape. Le pape n'est pas le seigneur des seigneurs : il n'est que le représentant du Seigneur sur terre (théocratie). L'accusation de féodalité, implicitement lancée par l'auteur, n'est donc pas rigoureusement fondée. Son rôle n'était certainement que de donner une certaine connotation médiévale, donc archaïque et antimoderniste, au gouvernement contemporain. Lamartine en parle comme d'un Ancien Régime pontifical, et sa critique est là.

Deuxièmement, dire du gouvernement pontifical qu'il est une aristocratie incarnée dans des "princes romains" est poser la question du rôle des laïcs dans un tel gouvernement. Selon D. Demarco, il y a à Rome « deux axes parallèles, le clergé et le laïcat. Le premier est formé du Collège des cardinaux, de la prélature et du bas-clergé, le second de l'aristocratie, de la bourgeoisie et du petit peuple. Au sommet [trônent] le Sacré Collège et l'aristocratie, au milieu la prélature et la bourgeoisie, à la base le peuple et le bas-clergé, séculier et régulier $\aleph^{22}$. A proprement parler, donc, l'aristocratie n'est pas dans le gouvernement pontifical, mais y est parallèle. Néanmoins, cet accompagnement du clergé par le laïcat le menace parfois d'une certaine ingérence. L'une des cinque piaghe della Santa Chiesa de Rosmini, en 1832 puis 1848, est la nomination des évêques : l'auteur propose leur élection par le clergé et les laïcs, et l'ouvrage, pour cette raison, sera mis à l'Index. Le gouvernement pontifical s'ouvrira progressivement aux laïcs et, en janvier 1848, le premier ministre laïc sera un prince : le prince Gabrielli, ministre delle $\mathrm{Armi}^{23}$. Si donc Lamartine a tort de faire, exagérément, du gouvernement pontifical lui-même une aristocratie, il n'a pas tort de souligner, par ce moyen, l'importance croissante que l'aristocratie laïque prendra pour le gouvernement pontifical en question.

\footnotetext{
${ }^{22}$ Domenico DEMARCO, «L'economia degli Stati italiani prima dell'unità », Rassegna storica del Risorgimento, 44, 1957, p. 255 ; cité et traduit par François JANKOWIAK, L'évolution des structures de la curie romaine [voir n. 21], p. 100.

${ }^{23}$ A la séance du 31 janvier 1848, Guizot répond à Thiers qu'il soutient la sécularisation d'une partie du gouvernement pontifical (La France parlementaire [voir n. 8], vol. 5, p. 560-561).
} 
JB Jeangène Vilmer, « Lamartine : les deux... », Revue d'Histoire Ecclésiastique, 2004

\section{2) UE gouverEemeEt élu (république et démocratie)}

La notion de république est ambiguë. On peut en distinguer deux sens. D'une part, un sens large et vieilli qui fait de « république » le synonyme d' «État», quel que soit ses formes. C'est la république de Bodin, par exemple, ou celle de Rousseau : tout État, tant qu'il est régi par des lois. D'autre part, un sens étroit et contemporain, qui désigne tout État qui n'est pas monarchique, c'est-à-dire le plus souvent tout État de type représentatif où la souveraineté est détenue par des représentants élus au suffrage universel. C'est d'ailleurs à propos des villes italiennes de constitution républicaine que le sens de « gouvernement républicain » du mot «république » apparaît pour la première fois en français dès 1410 . Notons que, même dans ce dernier cas, la définition reste encore très large : la république n'est pas tant une espèce de gouvernement particulier qu'un genre, susceptible d'englober plusieurs natures. Pour cette raison, l'utilisation qu'en fait Lamartine est problématique. Il fait de la république, au même titre que les autres (théocratie, oligarchie, monarchie, aristocratie, démocratie), une «nature de gouvernement» (1. 3), et non la nature de tous les gouvernements, ou même de certains d'entre eux. Il met sur le même plan, sur la même branche de l'arbre (de Porphyre), la partie et le tout, l'espèce et le genre : voilà qui est bien peu rigoureux. L'homme est poète, et non logicien. Cette notion, à vrai dire, est ambiguë depuis l'antiquité, et le plus logicien des grecs n'y échappe pas davantage : on trouve qu'à certains endroits ${ }^{24}$ Aristote distingue quatre $\pi \mathrm{o} \lambda \imath \varepsilon \varepsilon i ́ a 1$ : démocratie, oligarchie, aristocratie et monarchie ; mais qu'à

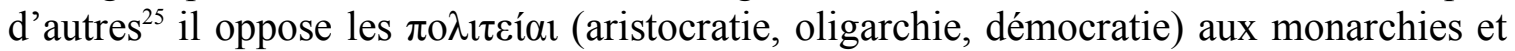
aux tyrannies. La république, tantôt intègre la monarchie, tantôt l'exclut : la source de sa polysémie se trouve à cet endroit.

Lamartine donne cependant une indication sur le sens qu'il attribue à " république » en précisant «car les chefs de l'oligarchie l'élisent». Le critère de la république serait donc l'élection, mais une élection particulière: celle effectuée par «les chefs de l'oligarchie », c'est-à-dire les cardinaux. « république » est ainsi entendue au sens étroit d'État de type représentatif où la souveraineté est détenue par des représentants élus - en l'occurrence élus par de " grands électeurs $»^{26}$ que sont les cardinaux.

Dans les faits, pourtant, il s'avère que le titre de république est parfaitement incompatible avec la nature du gouvernement temporel, dont le principe, nous l'avons vu, est monarchique tandis que la république entendue au sens étroit exclut précisément cette monarchie, même élective. C'est ce que rappellera Pie IX, le 15 novembre 1847, lors de la séance d'ouverture de la Consulta, lorsqu'il lancera à son égard cet avertissement déguisé, qu'en vertu de la continuité de la souveraineté le pape doit savoir refuser donc ne pas transmettre «toute institution incompatible avec la souveraineté pontificale $»^{27}:$ il visera là le principe même du gouvernement représentatif, vers lequel la Consulta s'efforcera d'évoluer pour gagner en autonomie. Il y aura finalement une preuve historique de l'incompatibilité de la république et de l'État pontifical, de leur

\footnotetext{
${ }^{24}$ Aristote, Rhétorique, I, 8, 1365b29-30 et Politique, IV, 7, 1293a38, par exemple.

${ }^{25}$ Aristote, Politique, III, 17, 1288a21 et V, 10, 1310a39, par exemple.

${ }^{26}$ Alphonse de LAMARTINE, article du 28 octobre 1847 [voir n. 8], p. 93.

${ }^{27}$ Cité par François JANKOWIAK, L'évolution des structures de la curie romaine [voir n. 21], p. 133, qui reprend et traduit David SILVAGNI, La Corte e la società romana nei XVIII e XIX secoli, Rome, 18841885,3, p. 421.
} 
contradiction, c'est-à-dire du fait que l'affirmation de l'un implique la négation de l'autre : l'abolition du pouvoir temporel par la république romaine le 8 février 1849. Mais l'expérience républicaine sera de courte durée: le 3 juillet, les troupes françaises investiront Rome et rétabliront l'autorité du pape Pie IX.

La démocratie est le régime politique dans lequel la souveraineté appartient à l'ensemble des citoyens, sans aucune distinction, c'est-à-dire au peuple $(\delta \eta \mu \mathrm{\varsigma})$. La définition de Lamartine est donc tout simplement fausse: n'est pas nécessairement une démocratie le régime dans lequel l'élection est le principe de la souveraineté. L'auteur omet effectivement un paramètre déterminant : encore faut-il que l'électeur en question soit le peuple lui-même. Or, en l'espèce, de quelle élection s'agit-il ? De celle du pape par les cardinaux («les chefs de l'oligarchie l'élisent»; «les cardinaux, grands électeurs ») et non par le peuple ! Il est certes question d'élection, mais pas de démocratie pour autant.

L'erreur de Lamartine a une vertu: celle d'insister, a contrario, sur le fait que le gouvernement pontifical n'est pas une démocratie, ce qui fut noté par d'autres en son temps. Rosmini écrivait déjà en 1832, c'est-à-dire au début du pontificat grégorien, que le bas clergé et les fidèles n'avaient aucun rôle en matière de nomination des évêques: quelle sorte de "démocratie » est-ce donc, si d'électeurs il n'y en a jamais que des «grands » $?^{28}$ Et les revendications républicaines, à la même époque, dénonçaient l'argument présenté par l'Église selon lequel la démocratie serait incompatible avec la papauté en tant que préture du Christ, c'est-à-dire avec le pouvoir spirituel : « on présente aux yeux des peuples un Ministère démocratique comme un véritable Antéchrist $»^{29}$.

\section{§2. UE « mécaEisme bizarre, compliqué, coEfus »}

Le gouvernement pontifical, dit Lamartine, a un «mécanisme bizarre, compliqué, confus $\|^{30}$. De ces trois adjectifs, on peut tirer deux vices : celui d'être complexe, c'est-àdire « compliqué » donc « confus » (la simplicité comme vertu), et celui, conséquent, d'être « bizarre », c'est-à-dire de ne pas être normal (la normalité comme vertu).

\section{A/ Le vice logique de la complexité : uEe juxtapositioE de coEtradictoires}

Lamartine écrit que le gouvernement pontifical a «les vices de toutes les natures de gouvernement, (...) réunis dans un seul gouvernement $»^{31}$. Cette phrase contient en elle deux critiques: le gouvernement pontifical a les vices de toutes les natures de gouvernement (critique explicite morale et politique) et ce faisant il a aussi le vice de réunir en un seul gouvernement toutes les natures des autres (critique implicite logique).

Cette seconde critique, implicite et logique, est à vrai dire la seule chose que l'auteur semble a priori avoir valablement démontré dans son texte. Le vice est en logique ce qui rend une pensée invalide. De ce point de vue, dire du gouvernement pontifical qu'il est vicié revient à le dire logiquement invalide, c'est-à-dire inconsistant, donc contradictoire.

\footnotetext{
${ }^{28}$ La question reste éminemment actuelle, adressée par exemple à la constitution américaine.

${ }^{29}$ Antoine JACQUEMOUD à G.-B. Michelini le 15 décembre 1848 [voir n. 16], p. 1165-1166.

${ }^{30}$ Lamartine, article du 28 octobre 1847 [voir n. 8], p. 93.

31 Ibid.
} 
Et c'est effectivement ce que s'efforce de montrer le passage précité, qui aligne ensemble des régimes politiques qui habituellement s'excluent. Le gouvernement pontifical, dit Lamartine, n'est pas l'un de ces régimes, il est « tout à la fois »: il est une chose et son contraire, il est en somme contradictoire. Pie VII, en 1816, ne disait pas autre chose, lorsqu'il écrivait que "l'unité et l'uniformité doivent être le fondement de toute institution politique [...]. Il manque encore à notre État cette uniformité, si utile aux intérêts publics comme privés, qui se sont formés de la réunion successive de différentes autorités, et présentent un agrégat d'usages, de lois, de privilèges, naturellement discordants entre eux, [et qui] rendent une province étrangère à l'autre, et qui divergent parfois, dans une même province, d'une zone à l'autre $»^{32}$.

A y bien regarder, pourtant, les régimes alignés peuvent parfaitement coexister en un gouvernement qui ne présentera pas l'intégralité de chacun, mais certains de ses aspects seulement. Rien, logiquement, ne s'oppose par exemple à ce que la monarchie, l'oligarchie et l'aristocratie coexistent: ils s'imbriquent comme trois cercles concentriques sans se gêner. Le développement de la centralisation et de l'absolutisme, ainsi, se généralise dans la plupart des autres États italiens jusqu'en 1870, sans pour autant chasser l'aristocratie : il s'agit plutôt d'une superposition. Plus généralement, individualité et collégialité ne sont pas forcément contradictoires. Cette stratification semble être essentielle à la méthode même de la Curie, comme le note F. Jankowiak, qui parle d'une tendance générale de la Curie romaine à « une superposition des structures par sédimentation $»^{33}$. C'est également ce que montre M. Pernot: "On forge des instruments nouveaux, mais on ne brise pas les anciens $»^{34}$. Si donc il fallait caractériser le mouvement de l'administration pontificale qui intègre ensemble des formes a priori contradictoires, en les absorbant sans souffrir de leur incompatibilité, il faudrait utiliser la dialectique hégélienne, d'ailleurs très en vogue à cette époque : par un mouvement de Aufhebung qui nie en intégrant, le gouvernement pontifical peut valablement être « tout à la fois ».

\section{B/ la vertu de la Eormalité : uEe critique du particularisme du gouverEemeEt poEtifical.}

Il n'est pas commun d'être « tout à la fois », d'avoir des aspects de chaque forme sans être intégralement aucune d'elles. Ce que le premier paragraphe du texte établit, en montrant la complexité du gouvernement pontifical, est son a-normalité, c'est-à-dire son caractère « bizarre ». Ce qu'il lui reproche est son étrangeté : on critique la déviance, le particularisme d'un corps - qui pour cette raison est peu et mal connu. Voilà qui, en cette matière, est très classique : c'est même, selon René Rémond, "l'élément premier, spontané ou réfléchi » qui est commun à toutes les formes d'anticléricalisme ${ }^{35}$. Reste que l'anticléricalisme qui s'attaque à la position des zelanti et au pouvoir temporel du pape n'est pas pour autant un anticatholicisme, car il croit malgré tout en un autre

\footnotetext{
${ }^{32}$ Pie VII, motu proprio Quanto per ammirabile du 6 juillet 1816, cité par François JANKOWIAK, L'évolution des structures de la curie romaine [voir n. 21], p. 77, qui reprend et traduit J. SPIZZICHINO, Magistrature dello Stato Pontifico (476-1870), Lanciano, 1930, p. 304, souligné par nous.

${ }^{33}$ François JANKOWIAK, L'évolution des structures de la curie romaine [voir n. 21], p. 117.

${ }^{34}$ Maurice PERNOT, Le Saint Siège : l'Église catholique et la politique mondiale, Paris, A. Colin, 1924, p. 42.

${ }^{35}$ René RÉMOND, L'anticléricalisme en France de 1815 à nos jours, Paris, Complexe, 1985, p. 20.
} 
catholicisme, réconcilié avec la modernité.

Que propose Lamartine ? Logiquement, de normaliser le gouvernement pontifical en lui attribuant, comme nous le verrons bientôt, une constitution. La réponse que fera, quelques mois plus tard, le jésuite G. Roothaan à I. A. de Pilat nous semble parfaitement applicable en l'espèce, puisqu'elle défend le droit de l'Église à l'originalité et refuse qu'elle doive s'aliéner sous la pression d'un « despotisme constitutionnel » : « Pour nous, la forme de gouvernement est indifférente. Mais la manière de le mettre en œuvre est la source des maux, et de fait rend les gouvernements constitutionnels autrement plus despotiques que ne le sont les gouvernements dits absolus ${ }^{36}$. La forme de gouvernement est indifférente : voilà qui répond à notre auteur. La normalité, en matière de régime politique, n'est pas un gage de vertu : tout dépend de la manière de gouverner et non de la nature du gouvernement lui-même.

\section{II- Un gouvernement vicié par l'instabilité}

L'introduction annonçait déjà « les vices de toutes les natures de gouvernement, sans leurs avantages (...)». Le premier paragraphe s'attachait à démontrer la première proposition (les vices de toutes les natures de gouvernement), sans y parvenir, à notre avis, puisqu'il ne suffit pas de montrer qu'un gouvernement a certains aspects de plusieurs autres pour en déduire qu'il hérite intégralement de tous leurs vices. Lamartine déduit, selon la règle de l'accessoire (l'accessoire suit le principal), que le gouvernement pontifical en héritant de toutes les natures de gouvernement, a nécessairement hérité de leurs vices. Mais il aurait fallu pour cela prouver que l'héritage était entier, et tout ce que l'on a montré, à vrai dire, est seulement que le gouvernement pontifical a certains aspects de certains des autres - pas même tous (pas la démocratie, par exemple, où l'erreur fut grossière). Il y a donc là un «saut» logique quelque peu rapide. Le second paragraphe, comme convenu, s'attache donc à démontrer la seconde proposition, à savoir qu'il n'hérite d'aucun de leurs avantages: "il n'a aucun de leurs bénéfices ». Ici encore, l'auteur se montre bien mauvais logicien. Voyons pourquoi.

Quand bien même le gouvernement pontifical aurait hérité de tous les vices des autres natures de gouvernement - ce qui en soi est contestable - rien ne serait susceptible de s'opposer à ce qu'il hérite pareillement de leurs bénéfices. N'est-il pas surprenant que, de la théocratie, l'oligarchie, la monarchie, la république, l'aristocratie et la démocratie, il ne retienne que les désavantages ? L'auteur sent bien la difficulté, et propose aussitôt d'en rendre raison: «C'est qu'à tous ces vices il ajoute le vice des vices en matière de gouvernement: l'instabilité $»^{37}$. Voilà donc l'argument: l'instabilité est ce tamis qui laisse passer les vices mais, curieusement, retient les vertus. Il n'y a rien là que d'arbitraire. Les lignes suivantes sont consacrées à développer cette instabilité, le passage étant franchi. Au regard de la logique, pourtant, il ne l'est pas : rien ne permet de comprendre que l'instabilité d'un gouvernement puisse traiter différemment les vices et les vertus. Soit sa traversée des autres natures de gouvernement est si instable qu'il n'en retient rien, et pas davantage les vices que les vertus, soit elle est suffisamment stable

\footnotetext{
${ }^{36}$ Lettre de G. ROOTHAAN à I. A. de Pilat du 4 mars 1848, citée et traduite par François JANKOWIAK, L'évolution des structures de la curie romaine [voir n. 21], p. 113.

${ }^{37}$ Alphonse de LAMARTINE, article du 28 octobre 1847 [voir n. 8], p. 93.
} 
pour qu'il en retienne quelque chose, et pas moins les vertus que les vices - à moins de présupposer que la vertu s'infuse lentement, tandis que le vice peut pénétrer et agir immédiatement, ce qui certes est poétique, mais bien peu valable en l'occurrence. Une fois de plus, l'écriture de Lamartine se montre peu rigoureuse.

Ceci étant dit, développons la nature de cette instabilité, dont l'auteur précise la teneur pour terminer.

\section{§1. L'iEstabilité daEs l'espace : uEe domiEatioE par l'étraEger}

Le gouvernement temporel de la papauté, par le biais de l'internationalisation du cardinalat, est dominé par l'étranger ${ }^{38}$ : c'est ce que Lamartine nous invite à développer ici. Pour mesurer l'importance de l'argument, il faut replacer notre extrait dans son contexte : il s'agit de la mineure d'un syllogisme destiné à montrer que l'on ne peut confier au pape le soin de confédérer l'Italie. Et la raison principale de cette méfiance n'est pas tant dans le «mécanisme bizarre, compliqué, confus » du gouvernement pontifical qu'en sa vulnérabilité qui, en l'espèce, est une porosité, une "domination par l'étranger » : une Italie confédérée par le pape serait une Italie aux mains de l'étranger. La suite le confirme : "Les cardinaux appartenant aux puissances rivales ennemies de l'Italie se concertent pour élire un chef qui leur soit docile ou vendu; la réaction contre le règne précédent commence avec le nouveau règne. Le Pape dépendant des puissances pour les intérêts de son Église comme pontife, est forcé d'en dépendre comme souverain italien. L'Italie confédérée contre les puissances aurait pour centre et pour chef de sa confédération l'allié obligé de ses ennemis $! »^{39}$. Une Italie aux mains de l'étranger, c'està-dire en d'autres mains que celles de la France, et voilà pourquoi il faut l'empêcher, quitte pour cela à proposer une intervention militaire - ce que le diplomate Lamartine s'empresse de faire, pour s'assurer, plus directement, du contrôle français en Italie. Si l'enjeu est grand, la question mérite une réponse: en quoi peut-on parler d'une « domination par l'étranger »?

Lamartine s'explique : les cardinaux élisent le pape, or ces cardinaux peuvent être étrangers donc étrangers aux intérêts de Rome, donc le pape peut être élu par l'étranger et contre l'intérêt de Rome. Il y a alors deux critiques: d'une part, celle qui vise la consistance interne d'un système dans lequel Rome élit contre l'intérêt de Rome (contradiction) et, d'autre part, celle qui vise le contrôle par l'étranger à proprement parler. Ce second point exige quelques développements.

Premièrement, Lamartine limite la question aux cardinaux. Le problème est alors de savoir quelle est la proportion d'étrangers dans la composition du Sacré Collège. La période à laquelle Lamartine écrit, en 1847, correspond effectivement à un changement très net dans l'ouverture du Sacré Collège à l'étranger : alors qu'à l'origine la domination italienne était très forte (environ $80 \%$ de nationaux depuis le XIVe siècle, et encore $68 \%$ issus de 1'État pontifical lui-même sous Grégoire XVI) ${ }^{40}$, elle décroît spectaculairement

\footnotetext{
${ }^{38}$ En 1860, l'auteur présentera également l'argument réciproque et complémentaire : son gouvernement, spirituel cette fois, est une domination des pouvoirs temporels étrangers catholiques (Cours Familier [voir n. 11], vol. 9, 1860, p. 447).

${ }^{39}$ Alphonse de LAMARTINE, article du 28 octobre 1847 [voir n. 8], p. 94.

${ }^{40}$ Selon Philippe BOUTRY, La Restauration de Rome. Sacralité de la ville, tradition des croyances et recomposition de la Curie à l'âge de Léon XII et de Grégoire XVI (1814-1846), thèse pour le doctorat d’État, université Paris-IV Sorbonne, 1994, vol. 2, p. 248-249.
} 
sous le règne de Pie IX, élu en 1846, pour atteindre 58\%. L'internationalisation du cardinalat s'avère être proportionnelle à la perte d'influence romaine qu'elle est sensée compenser. Ce que l'auteur dénonce en 1847 est donc bel et bien une tendance qui se confirmera dans les années suivantes.

Cette ouverture à l'étranger, cependant, devrait en principe se trouver limitée par une autre ouverture qui lui est contemporaine : celle aux laïcs. Car tant que l'écrasante majorité des hauts postes de l'administration et de la diplomatie au sein du gouvernement pontifical était réservée aux ecclésiastiques, la nature cosmopolite et la dimension internationale de l'Église charriait naturellement à ces postes des non nationaux, comme le montre Leopoldo Galeotti dans Della sovranità e del governo temporale dei Papi, publié à Lausanne en 1847, c'est-à-dire l'année même où Lamartine rédige son texte. Mais avec l'ouverture de ces postes aux laïcs, le gouvernement peut puiser davantage dans le réservoir local, c'est-à-dire national, et limiter à son gré le recours à l'extérieur. Notons que l'utilisation lamartinienne de l'internationalisme de l'Église prend le contrepied de celle qu'en fait habituellement l'anticléricalisme, pour qui l'Église est une puissance internationale qui dénationalise ses fidèles. Ce que dit Lamartine est que les premiers à être «dénationalisés », en l'occurrence, sont les Italiens eux-mêmes. L'internationalisme de l'Église est une force qui, lancée hors de Rome, lui revient pour frapper sa souveraineté, c'est-à-dire son indépendance.

Deuxièmement, y a-t-il effectivement une ingérence étrangère dans l'élection des pontifes? Les intrigues diplomatiques autour des élections pontificales, en effet, ne manquent pas. En octobre 1833, quinze ans avant Lamartine, C. Didier résumait déjà sur un ton très satirique dans la Revue de Paris nombre des reproches qui sont ici adressés (des intrigues, une élection, l'intervention étrangère $)^{41}$. On sait par exemple que si l'élection de Grégoire XVI dura si longtemps ( $1^{\mathrm{er}}$ décembre $1830-2$ février 1831$)$, c'est que von Metternich faisait pression sur les cardinaux pour obtenir un pape favorable à la Sainte-Alliance. De la même manière, c'est la pression espagnole qui avait facilité l'élection de Sixte Quint le 24 avril 1585, contre les résistances de certains membres du conclave - et Lamartine cette fois ne s'en plaint pas. L'ingérence étrangère dans les élections pontificales pose effectivement la question de la liberté et de la sécurité de l'élection, une question que la papauté se montre soucieuse de résoudre. Il faudra attendre près d'un quart de siècle, cependant, pour que des documents pontificaux définissent, en 1871, les conditions de légitimité de l'élection du pape.

Troisièmement, l'ingérence étrangère déborde la question des seules élections : il s'agit, plus largement, de toutes les interventions armées sur le territoire italien, à la demande des papes. Il se pourrait fort que l'auteur pense ici à la répression sanglante de l'insurrection libérale dans les États pontificaux en 1831, à l'occasion de laquelle Grégoire XVI avait sollicité une intervention autrichienne. Cet épisode avait à l'époque compté pour Lamartine, comme en témoigne ce que l'on pourrait nommer «l'affaire de l'Ode deuxième à Némésis ». La répression sanglante ordonnée par Grégoire XVI avait été condamnée en France dans certains milieux, notamment par Hugo dans ses Feuilles d'Automne, XL, et par Barthélémy qui publie dans l'hebdomadaire satirique Némésis, le 12 février 1831, une ode intitulée Au Pape dans laquelle il s'en prend à Grégoire XVI. Lamartine, le 20 février, rédige alors une réponse, Rome, ode deuxième à Némésis, pour défendre le pape, mais ne la publie finalement pas quand il apprend que Casimir Périer

\footnotetext{
${ }^{41}$ Voir C. DIDIER, « Un conclave », Revue de Paris, t. 55, 1833, p. 8 et 12.
} 
débarque le 22 février à Ancône pour s'opposer à l'avancée des troupes autrichiennes dans les États pontificaux. Il conservera néanmoins son texte, et l'utilisera cinq ans plus tard pour composer une partie de la neuvième époque de Jocelyn, publié en $1836^{42}$.

Quatrièmement, enfin, Lamartine lui-même notera en 1860 que la domination par l'étranger du pouvoir temporel de la papauté n'est autre que la raison d'être et l'origine du pouvoir temporel lui-même. Le pouvoir temporel du pape constitue en effet pour les puissances étrangères la seule prise et, par conséquent, le seul contrepoids possible face à la domination papale du pouvoir spirituel. On a donc donné au pape, déjà spirituellement cosouverain des États catholiques, une responsabilité temporelle, « un gage humain dans une possession territoriale quelconque, responsabilité et gage par lesquels nous puissions le modérer, le saisir et le punir temporellement comme prince, s'il viole envers nous les limites de son droit comme pontife $»^{43}$.

\section{§2. L'iEstabilité daEs le temps : le problème de la coEtiEuité du pouvoir}

\section{A/ L'iEstabilité daEs le temps}

" Je ne veux pas d'un monde où tout change, où tout passe; Où, jusqu'au souvenir, tout s'use et tout s'efface; Où tout est fugitif, périssable, incertain ; Où le jour du bonheur n'a pas de lendemain!» ${ }^{44}$

Lamartine justifie son accusation d' « instabilité » par une énumération : "Il est temporaire, il est court, il est précaire, il est fugitif $»^{45}$. Encore une fois, les mots sont importants : les deux premiers témoignent de cette instabilité comme d'un état de fait, tandis que les deux autres impliquent déjà la cause d'un tel état.

\section{1) La maEifestatioE de l'iEstabilité : uE poEtificat « temporaire » et « court»}

Le pape est élu à vie, le pontificat est viager. Dire qu'il est court est donc dire que la vie du pape sur le Siège est courte. Pour quelle raison? Lamartine s'explique : on « élit un vieillard $»^{46}$. Déjà, dans Jocelyn, on pouvait lire ces vers :

\footnotetext{
«Quand deux fois en dix ans les Gaulois, dans la poudre, Ont par leurs cheveux blancs traînés ces dieux sans foudre, Et mis le temple à nud et l'autel à l'encan, Et que de ces vieillards, qu'outrage encore la haine, L'un mourut sans tombeau, l'autre possède à peine L'ombre courte du Vatican ! ${ }^{47}$
}

\footnotetext{
${ }^{42}$ Sur l'affaire de l'Ode deuxième à Némésis, on peut voir «Lamartine défend le pape et se ravise », in Henri GUILLEMIN, Connaissance de Lamartine, Fribourg, Librairie de l'université, 1942, p. 157-186.

${ }^{43}$ Alphonse de LAMARTINE, Cours Familier [voir n. 11], vol. 9, 1860, p. 447.

${ }^{44}$ Alphonse de LAMARTINE, La Foi, Méditations poétiques, in Euvres poétiques complètes, MariusFrançois Guyard (ed.), Paris, Gallimard, 1963, p. 50.

${ }_{45}^{45}$ Alphonse de LAMARTINE, article du 28 octobre 1847 [voir n. 8], p. 94.

${ }^{46}$ Ibid.

${ }^{47}$ Lamartine, Jocelyn, Neuvième époque, in CEuvres poétiques complètes [voir n. 44], p. 752-754.
} 
La critique est commune, et d'autres en France, dans la vague d'anticléricalisme soulevée par la révolution de Juillet, ont noté l'âge avancé des souverains pontifes : «L'élection perpétue au Vatican une hérédité d'impuissance et de sénilité » écrit E. Pelletan dans la Nouvelle Minerve du 2 octobre 1836; tandis que Chateaubriand, en décembre 1831, se souvenait de ce Jeudi-Saint de 1829 où il avait vu « le pauvre vieux Pape paralytique ${ }^{48}$. Lamartine pense sans doute à l'exemple de Pie VIII qui, en 1829, succède à Léon XII alors qu'il est déjà un vieillard aux forces mourantes : son pontificat ne durera que vingt mois. Celui de Léon XII (1823-1829) ne fut pas très long non plus. Force est de constater, effectivement, que depuis sa maturité jusqu'en 1847, Lamartine n'a pu observer que des pontificats mouvementés, passagers et momentanés. Il ignore qu'il est à une période charnière, qui marque le début d'un nouveau cycle, fort stable cette fois, puisque Pie IX et son successeur Léon XIII atteindront et dépasseront ce que l'on nomme les « années de Pierre », c'est-à-dire les vingt-cinq années de pontificat.

\section{2) La cause de l'iEstabilité : uE gouverEemeEt « précaire » et « fugitif », coEtrôlé par l'oligarchie des cardiEaux}

«Précaire » est un mot riche de sens. Le latin precarius, du verbe precari (prier, supplier), désigne ce qui est obtenu par prière. Le droit prolongera cette définition en utilisant « précaire » pour ce qui n'existe ou ne s'exerce que par une permission toujours révocable. Dire du gouvernement pontifical qu'il est précaire est donc dire qu'il est contingent, c'est-à-dire qu'il peut ne pas être, qu'il est révocable à tout moment. Dire de lui, ensuite, qu'il est " fugitif », est le dire menacé, car l'on fuit face à un danger. Pour ces deux raisons, il n'offre par conséquent aucune garantie de durée, de stabilité donc de solidité. La question qui se pose d'emblée est alors la suivante : si le pontificat n'existe et ne s'exerce que par une permission toujours révocable, qui est l'auteur de cette permission? S'il fuit, qui le menace?

Lamartine y répond: "l'oligarchie électorale des cardinaux, pressée comme dit l'historien romain, de voir finir et dévorer le règne d'un moment $(..) »^{49}$. La cause de l'instabilité se trouve donc là : elle est voulue et organisée par l'oligarchie des cardinaux, pour mieux contrôler le pape et limiter sa monarchie. Nous avions vu comment l'oligarchie cardinalice était l'une des limitations de l'absoluité de la monarchie pontificale : la voici en action. Quels sont ses moyens ? L'auteur en donne deux : elle "élit un vieillard » d'une part, nous l'avions déjà noté, et elle « choisit une main débile pour avoir à la décharger plus tôt du poids du sceptre $»^{50}$.

Résumons : l'oligarchie des cardinaux s'assure le contrôle du gouvernement en élisant un vieillard, aisément remplaçable, ou un pape faible, aisément manipulable. Mais qu'advient-il lorsque le pape est fort? Les cardinaux patientent, car ils savent que sa puissance mourra avec lui : " dans tous les cas les pensées du Pape fort meurent avec lui $»^{51}$. Et avec la nouvelle élection ils retrouveront leur pouvoir.

Derrière ce constat se dessine un reproche : au gouvernement pontifical il manque la

\footnotetext{
${ }^{48}$ Revue Européenne, t. II, p. 4.

${ }^{49}$ Alphonse de LAMARTINE, article du 28 octobre 1847 [voir n. 8], p. 94.

50 ibid.

51 ibid.
} 
JB Jeangène Vilmer, « Lamartine : les deux... », Revue d'Histoire Ecclésiastique, 2004

continuité. C'est ce qu'il nous faut maintenant développer.

\section{B/ Le problème de la coEtiEuité du pouvoir daEs le gouverEemeEt poEtifical}

« Il n'y a pas d'hérédité ni de survivance de système ni de constitution garantissant l'avenir dans un tel gouvernement; le Pape d'hier n'engage pas celui de demain. Les œuvres y sont personnelles, la vertu y est viagère $»^{52}$. Dans ce dernier morceau est clairement dénoncé le problème de la continuité dans le gouvernement pontifical. Mais l'auteur ne se contente pas de dénoncer : par là même et implicitement il propose. Nous en rendrons compte en deux temps.

\section{1) « le pape d'hier E'eEgage pas celui de demaiE» : répoEse à l'objectioE}

Lamartine reprend à son compte le proverbe romain suivant : « la regola di ogni Papa é il rovescio di quella del suo predecessore » (la règle de tout pape est le renversement de celle de son prédécesseur). Pourtant, l'affaire n'est pas si simple.

Le problème de la continuité est au cœur d'un certain paradoxe. Nous avons vu comment la tendance structurelle de la Curie était à la juxtaposition, la superposition, la stratification, des différents outils accumulés au cours du temps; comment, de cette manière, elle pouvait intégrer de manière dialectique des formes politiques a priori incompatibles. N'était-ce pas déjà la manifestation d'une certaine continuité ? Le fait de nier en intégrant, et non en écrasant, qui définit proprement le mouvement dialectique, signifie à lui seul la continuité de la Curie romaine. Comment, dès lors, comprendre que « le Pape d'hier n'engage pas celui de demain »? De notre point de vue, et si la vision dialectique de l'histoire de la Curie romaine est juste, le Pape d'hier n'engage certes pas celui de demain en tout, mais celui de demain ne pourra nier celui d'hier qu'en l'intégrant, c'est-à-dire en construisant sur ses acquis.

Qu'on s'attache d'ailleurs à l'argument suivant. Que «le pape d'hier n'engage pas celui de demain », est-ce en soi un mal ? Lamartine prend les exemples de papes exceptionnellement compétents, Sixte-Quint et Pie IX. Il avoue même dans les lignes suivantes de son article qu'il s'agit là de cas fort rares : «Pie IX est un hasard, ou plutôt une providence ; mais Pie IX est un coup du sort. Il faut le saisir comme on saisit un coup du sort, l'heureuse fortune de l'Italie, en remerciant le ciel, mais sans y compter deux fois $! \nu^{53}$. Mais qu'arriverait-il dans le cas d'un pape exceptionnellement incompétent, si le Siège en gardait trop la mémoire ? Lamartine suggère-t-il que Grégoire XVI aurait dû imprimer davantage son temps ? Suggère-t-il que le pontificat grégorien engage celui de Pie IX? Certes non! Notre auteur se montre au contraire fort satisfait que le nouvel élu ne soit pas enchaîné à l'ancien et qu'il ait les mains libres pour réformer un gouvernement qui en a besoin.

Deuxième limitation à l'absoluité de la monarchie pontificale: la tradition. La tradition et les institutions assurent un contrepoids suffisamment lourd pour relativiser

\footnotetext{
${ }^{52}$ ibid.

${ }^{53}$ Ibid.
} 
l'importance de la personne même du pape ${ }^{54}$. Et ce mécanisme agit effectivement comme une protection contre la qualité relative et aléatoire des papes qui se succèdent sur le Siège.

Ainsi la continuité n'est-elle pas absente du gouvernement pontifical. Ce qui engage le pape de demain n'est certes pas celui d'hier, mais l'ensemble des institutions et le poids de la tradition. Le pape de demain, d'ailleurs, peut lui-même s'inspirer de papes d'hier : ainsi Pie IX se place-t-il dans la continuité de l'exemple de Pie VI et Pie VII, dont il reprend le nom. Ce n'est pas dire que Lamartine a tort, que l'instabilité n'est pas un problème. Assurément elle l'est, nous n'écrivons pas « le pape est mort, vive le pape ! ». Mais il convenait de relativiser cette question, avant d'étudier, à présent, la réponse de l'auteur.

\section{2) Le mouvemeEt coEstitutioEEaliste daEs les États de l'Église et l'iEflueEce de l'expérieEce révolutioEEaire et coEstitutioEEelle fraEçaise (1847-1848)}

Dans l'énumération «pas d'hérédité ni de survivance de système ni de constitution », il ne faut pas mettre tous les termes sur le même plan. Les deux premiers ne sont que des genres. Lamartine ne suggère pas, effectivement, de remplacer la monarchie élective par une monarchie héréditaire classique, ce serait grotesque au regard des dogmes les plus élémentaires de l'Église. De la même manière, par "survivance de système » il est suffisamment vague pour ne rien désigner: hérédité et survivance sont pris comme synonymes de continuité. Ces deux premiers termes, donc, désignent la fin à atteindre. Le troisième, par contre, est le moyen: le meilleur moyen de garantir la survivance du système, son "hérédité » au sens figuré, est de le constitutionnaliser - voilà ce que propose notre auteur. Il s'agit alors de savoir, comme l'écrira Ch. Van Duerm, «si le royaume pontifical était mûr pour cet esprit de liberté et de progrès divers, qui envahissait le monde moderne $(\ldots) \|^{55}$.

Le royaume pontifical, en 1847, est mûr : il est à point, et la France ne l'ignore pas. Le 29 janvier 1848, à la chambre des députés, Guizot est interrompu : « On veut se servir du pape pour le remaniement de l'Italie, et pour une organisation politique bien près d'être républicaine. - Constitutionnelle! [l'interrompt M. Glais-Bizoin]. - Il ne s'agit pas du tout de constitution à l'heure qu'il est! De quoi il s'agira dans dix ans, dans vingt ans, je ne le sais pas; je ne suis pas obligé de traiter aujourd'hui à cette tribune les questions que nos successeurs y traiteront. Je traite les questions actuelles. Or, quant à présent, il ne s'agit pas de constitutions dans les États italiens $(\ldots) »^{56}$. Guizot se montre prudent et rassurant, mais il a tort : c'est bien de constitutions qu'il s'agit déjà dans les États italiens.

Les États de l'Église vivent en effet une effervescence constitutionnaliste sans précédent. Pellegrino Rossi, qui en est le chef de file, est également depuis mai 1846 l'ambassadeur du gouvernement pontifical en France. Lamartine le connaît

\footnotetext{
${ }^{54}$ Voir Maurice PERNOT, Le Saint Siège [voir n. 34], p. 205.

${ }^{55}$ Charles VAN DUERM, Vicissitudes politiques du pouvoir temporel des papes de 1790 à nos jours, Lille, Desclée De Brouwer et Cie, 1890, p. 172.

${ }^{56}$ Discours de François GUIZOT à la chambre des députés, 29 janvier 1848, in Histoire parlementaire de France, recueil complet des discours prononcés dans les Chambres de 1819 à 1948, t. V, Paris, MichelLévy frères, 1863-1864, p. 551.
} 
personnellement. En 1842, V. de Mars écrivait déjà : «C'est vers les gouvernements constitutionnels que se portent l'opinion publique, le vœu et l'espérance des nations. C'est auprès des gouvernements constitutionnels que le catholicisme trouve respect, justice, protection. (...) l'avenir de Rome est là, dans son alliance intime avec les gouvernements constitutionnels $\gg{ }^{57}$. Le modèle est même précis : il s'agit de la France, et de sa Charte de 1830, qui est conçue comme un pacte, un contrat (J.-C. Caron et M. Vernus parlent de «lien contractuel entre le souverain et la nation » ${ }^{58}$ ). En 1831 déjà, la révolution parisienne avait provoqué des remous sur le versant italien : des insurrections à Modène, Parme, dans les Marches, et en Romagne. Les révolutionnaires des États pontificaux avaient alors proclamé l'abolition du pouvoir temporel des papes. Voilà qu'à présent, toujours sur le modèle français de 1830, c'est la constitution qu'ils reprennent. Lorsque Stendhal, en 1817 déjà, écrivait que l'Italie « (...) a dans le sang trois siècles de despotisme, il lui faut des maîtres constitutionnels $\rangle^{59}$, nul doute que c'est à la France, et à son expérience en matière de constitutions, qu'il pensait également.

Dans les années 1846-1848, le mouvement constitutionnaliste s'accélère et un bloc de constitutionnalité se construit en intégrant de plus en plus de textes qui, conformément au modèle français, sont des pactes: leur origine est mixte, ni seulement populaire ni seulement princière. Nous annoncions tout à l'heure le mouvement constitutionnaliste comme l'une des limitations de l'absoluité de la monarchie pontificale : les textes se présentent effectivement comme une autolimitation du souverain. Ainsi en est-il, par exemple, de la décentralisation introduite par le motu proprio du 2 octobre 1847 sur le Municipio di Roma : la plupart des responsabilités étaient jusqu'alors centralisées par un gouvernement qui, par nature, est titulaire des trois pouvoirs, distincts seulement virtuellement: " le souverain pontife et les évêques sont, tout à la fois, législateurs, administrateurs et juges $\rangle^{60}$. Avec la réforme, certaines d'entre elles sont attribuées par le gouvernement central à l'administration municipale. Le contrôle du pape sur les décisions municipales est également allégé : il ne s'agit plus d'un contrôle d'opportunité qui lui permettait d'annuler purement et simplement toute décision municipale, le pape ne contrôle plus désormais que la légalité des décisions.

Lamartine fait paraître son article le 28 octobre 1847. Cette même année, la tendance constitutionaliste qui agite le gouvernement pontifical s'est déjà manifestée à travers la création très récente de deux institutions. Le Conseil des Ministres, d'une part, créé par le motu proprio du 12 juin 1847 (et redéfini par celui du 29 décembre 1847) était composé de trois cardinaux (secrétaire d'État, camerlingue et préfet des Eaux et routes), et de quatre prélats (Auditeur de la Chambre, gouverneur de Rome, trésorier général et président des armées). Son importance, cependant, est relativisée par la concurrence dans laquelle il se trouve avec la congrégation des Affaires ecclésiastiques extraordinaires : il n'y a pas un organe exécutif majeur dans l'État pontifical. La Consulta di Stato, d'autre part, était prévue dès le 19 avril 1847 mais fut créée par le motu proprio du 14 octobre 1847, soit deux semaines seulement avant la parution de l'article de Lamartine. Comme

\footnotetext{
${ }^{57}$ V. DE MARS, «Chronique de la Quinzaine, 31 octobre 1842 », Revue des Deux Mondes, quatrième série, tome XXXII, octobre-décembre 1842, p. 524.

58 Jean-Claude CARON et Michel VERNUS, L'Europe au XIXe siècle. Des nations aux nationalismes, 1815-1914, Paris, A. Colin, 1996, p. 50.

${ }^{59}$ STENDHAL, 18 juillet 1817, Voyages en Italie, V. Del Litto (ed.), Paris, Gallimard, 1973, p. 146. C'est en l'occurrence une monarchie parlementaire à l'anglaise qu'il suggère.

${ }^{60}$ Raoul NAZ (dir.), Dictionnaire de droit canonique, Paris, Librairie Letouzey et Ané, 1935, p. 191.
} 
le note F. Jankowiak, " la Consulta représentait certes une évolution, bien qu'il soit difficile d'y voir un signe de laïcisation de l'appareil d'État; elle apparaît davantage comme l'adjonction d'un organisme laïc consultatif à la structure centrale ecclésiastique, seule investie du pouvoir décisionnel, dont la Consulta dépendait en tant qu'instance auxiliaire $\rangle^{61}$. C'est précisément dans le cadre d'une volonté d'émancipation et d'évolution vers un parlementarisme que l'on débattra durant l'hiver 1847-1848 de la question de la publicité des votes de la Consulta, qui sera finalement proclamée, à l'exception des élections internes. Elle devient de fait un organe délibérant.

L'actualité constitutionnelle est donc chargée de l'autre côté des Alpes. Aussi comprend-on que, lorsque Lamartine publie ses réflexions sur la nature du gouvernement pontifical, l'évocation finale d'un «constitution » n'a rien d'hasardeuse : elle semble annoncer son arrivée prochaine, comme l'aboutissement d'une tendance dont il n'ignorait rien. Il l'appelle, y prépare, met mieux en évidence sa nécessité. Tout ce qu'il souhaite à son texte, en somme, c'est-à-dire à son accusation d'instabilité, est de se périmer au plus vite. La constitution qu'il attendait arrivera quelques mois plus tard et, du fauteuil de Guizot qu'il occupera alors, Lamartine, on le devine, pensera à son article d'octobre : le Statuto du 14 mars 1848, qui s'inscrit dans une vague déferlante sur les États italiens (Ferdinand II avait promulgué une constitution le 29 janvier, Léopold de Toscane le 11 février, et Charles-Albert le 5 mars), marquera nettement une ligne de séparation entre les pouvoirs spirituels, immuables, et temporels, susceptibles d'évoluer avec la société. Le Sacré Collège deviendra, au sens contemporain cette fois, le «Sénat de l'Église ».

Les thèses défendues dans cet article du 28 octobre 1847, notre auteur les reprendra à partir de janvier 1848 pour sa rentrée parlementaire, dans des débats qui l'opposeront à Guizot sur la question italienne. Victor Hugo, intervenu le 12 janvier en insistant sur le rôle du Pape, reconnaîtra le 28 avoir "manqué » son sujet par rapport au «magnifique discours » de Lamartine. L'homme, effectivement, est convaincant. Par le verbe il écarte plus d'un adversaire.

Dans notre extrait, c'est peu dire qu'il ne manque pas de qualité littéraire. La rhétorique est belle et puissante, le choix des mots est habile, et les idées s'enchaînent harmonieusement. Le raisonnement, en apparence, semble clair: deux parties développent ce que l'introduction promet. On a vu, cependant, le vernis se craqueler, sous les coups de butoir d'une lecture exigeante : la thèse annoncée n'est pas démontrée, l'auteur par deux fois prend avec la déduction une certaine liberté étrangère à la logique. Seul un lecteur pressé, naïf et peu attentif, se laissera ainsi convaincre que le gouvernement pontifical a les vices de toutes les natures de gouvernement et, curieusement, aucun de leurs bénéfices; qu'il est une "démocratie » par le seul fait que l'élection en est le principe ; et qu'il faut déplorer que le pape d'hier n'engage pas celui de demain, même si celui d'hier était un zelanti sordide quand celui de demain s'annonce salvateur. Certaines de ces thèses défient le bon sens, d'autres les lois de l'inférence logique, d'autres, enfin, notamment dans le premier paragraphe, partent de définitions tout simplement erronées. Lamartine, finalement, s'est montré plus grand orateur que philosophe.

\footnotetext{
${ }^{61}$ François JANKOWIAK, L'évolution des structures de la curie romaine [voir n. 21], p. 129.
} 
Il est cependant un mérite que l'on doit sans rougir reconnaître à l'auteur : celui d'avoir glissé, volontairement peut-être, entre les lignes de ces réflexions italiennes, des considérations très françaises. Nous avons vu comment, par la situation même de cet extrait dans l'article du 28 octobre 1847, Lamartine, en diplomate, proposait l'appui de la France pour confédérer l'Italie contre la solution néo-guelfe. Comprenons maintenant que la critique même exposée par le texte, celle d'un gouvernement vicié par sa complexité et son instabilité, vise peut-être moins l'État de l'Église que l'État français : il nous semble que l'auteur, dans ces lignes, se sert du gouvernement pontifical comme d'un miroir.

Qu'en est-il, effectivement, de la situation française? Elle est complexe. Le gouvernement, lui aussi, est «tout à la fois»: initialement portée au pouvoir dans l'optique d'une monarchie limitée à l'anglaise, la Monarchie de Juillet a dans les faits l'apparence d'une oligarchie, autour de Louis-Philippe et de Guizot, dont les théoriciens (Thiers, Guizot et les autres) sont hostiles à la démocratie pure comme à la monarchie pure, c'est-à-dire absolue. Le temps est donc à la nuance, au compromis entre les différents régimes, plutôt qu'au choix de l'une des formes pures. Le climat politique de l'époque est celui du mélange. Le mécanisme, en somme, est bel et bien «bizarre, compliqué, confus ».

La situation française n'est pas seulement complexe : elle est aussi instable. En 1847, l'avenir de la Monarchie de Juillet est «temporaire, court, précaire et fugitif». La révolution approche, en France comme en Italie. Et, d'un côté comme de l'autre, le nouveau gouvernement s'annonce déjà seulement «provisoire ». De l'autre côté des Alpes, la situation est instable : l'insurrection de Palerme du 2 janvier et le ralliement du roi de Piémont à un programme libéral par le Statut constitutionnel du 5 mars échauffent les esprits. L'Italie est en effervescence, des révolutions éclatent, puis déclinent. Le Risorgimento révolutionnaire est un échec. Rossi, rentré au pays pour devenir Premier Ministre de Pie IX, sera assassiné par un démocrate le 15 novembre 1848 et, quelques mois plus tard, l'expérience républicaine sera close et le gouvernement pontifical rétabli.

Le parallélisme des situations est donc frappant : c'est précisément dans la complexité et l'instabilité, les deux axes de notre texte, que la France et l'Italie à l'époque se rejoignent. En l'espèce, c'est de deux grandeurs déchues dont il s'agit: celle des ambitions étrangères de la France, brisées par le Congrès de Vienne de 1815, et celle de la papauté, mise à mal par Napoléon en 1814, et qui tente désormais de retrouver sa puissance et son prestige. On peut sans peine supposer que cette double lecture n'est pas une fantaisie de commentateur mais bien une ruse d'auteur. La nature du gouvernement pontifical n'intéresse guère en elle-même les lecteurs du Bien public, et la critique de Lamartine, nous l'avons vu dans l'introduction, ne porte pas tant sur le gouvernement luimême que sur sa capacité à confédérer une Italie éclatée et en ébullition. Pour le bien public, précisément, l'auteur défend là une position utile à la France : il la renseigne sur sa voisine, en tire une liste de problèmes, et propose des solutions. Mais il y a plus : par là même il lui rappelle que ses propres problèmes sont les mêmes, et que les solutions en question, par conséquent, lui sont également applicables. La France et l'Italie sont complexes et instables : il leur faut des constitutions. Voilà ce qui dit l'auteur, et ce n'est pas son moindre talent que d'être à ce point visionnaire. 